\title{
PERFORMANCE OF EXTENDED SPACE-TIME CODING TECHNIQUES FOR MIMO MC-CDMA SYSTEMS
}

\author{
Joaquim Bastos ${ }^{(1)}$, Atílio Gameiro ${ }^{(2)}$ \\ (I) Instituto de Telecomunicações, Campus Universitário de Santiago, 3810 Aveiro, Portugal,Email: jbastos@av.it.pt \\ (2) Universidade de Aveiro, Campus Universitário de Santiago, 3810 Aveiro, Portugal, Email: amg@det.ua.pt
}

\begin{abstract}
In this paper we consider a transmission system based on MC-CDMA, where signal spreading is performed entirely in the frequency domain. An extended spacetime coding technique, the Double Alamouti, is evaluated considering a MIMO channel. This assessment is made against standard Alamouti coding, for two different $\mathrm{Tx} / \mathrm{Rx}$ antenna schemes $(2 \times 1$ and $2 \times 2)$. Numerical results, attained through system model simulations, are presented for performance evaluation under realistic scenarios considering some typical system impairments. These results show that in practical systems significant improvements can be achieved by using the Double Alamouti coding scheme.
\end{abstract}

\section{INTRODUCTION}

It is rather consensual that the provision of broadband capabilities in wireless communication systems will have to resort to multiple transmit and receive antennas in order to exploit the rich scattering properties of wireless propagation channels (MIMO, Multiple Input Multiple Output).

On the other hand multicarrier code-division multiple access (MC-CDMA) has been considered for the past few years by many researchers to be a very promising candidate as the access scheme for Beyond $3 \mathrm{G}$ mobile communications systems [1][2]. This technique benefits from the features of Orthogonal Frequency Division Multiplexing (OFDM) and CDMA. One important aspect of this technique is the fact that it takes advantage of frequency diversity since signal spreading is performed purely in the frequency domain, making possible to use diversity combining based techniques for signal detection [3].

To also explore space and time diversity in order to help mitigate channel's frequency selective fading, STBC (Space-Time Block Codes) have been proposed [4]. One of the simplest schemes is the Alamouti space-time code [5] which does not require very complex decoders and can be integrated in the existing systems without needing much redesign. The basic Alamouti scheme based on $2 \times 1$ (two transmit and one receive antennas) can be extended by using multiple Alamouti encoders in parallel. The advantage of using a coding scheme based on multiple Alamouti is that it is possible to lower the modulation constellation size used in the system while maintaining the same data rate and bandwidth. This in turn will allow better tolerance to amplitude or phase errors caused by automatic gain control (AGC) or radiofrequency $(\mathrm{RF})$ circuits. In this communication we consider the use of a Double Alamouti coding scheme (4x2). For that, we also designed a space-time combiner, including equalization, and compare its performance against the conventional 2x1 Alamouti scheme, using spatial channel models proposed for B3G systems. A $2 \times 2$ scheme, implementing the Alamouti code, based on spatial combination at reception is also considered for comparison purposes.

This paper is organized as follows. Section 2 provides the description of the considered system model. In section 3 all implemented space-time coding schemes are presented. Illustrative simulation results are presented and analyzed in section 4, and finally the main conclusions are outlined in section 5 .

\section{SYSTEM MODEL}

In this paper we consider the downlink of a multiuser communication system based on MC-CDMA. In order to evaluate system's performance a simulation chain was implemented in software. The structure of that transmission chain is represented in Fig. 1.

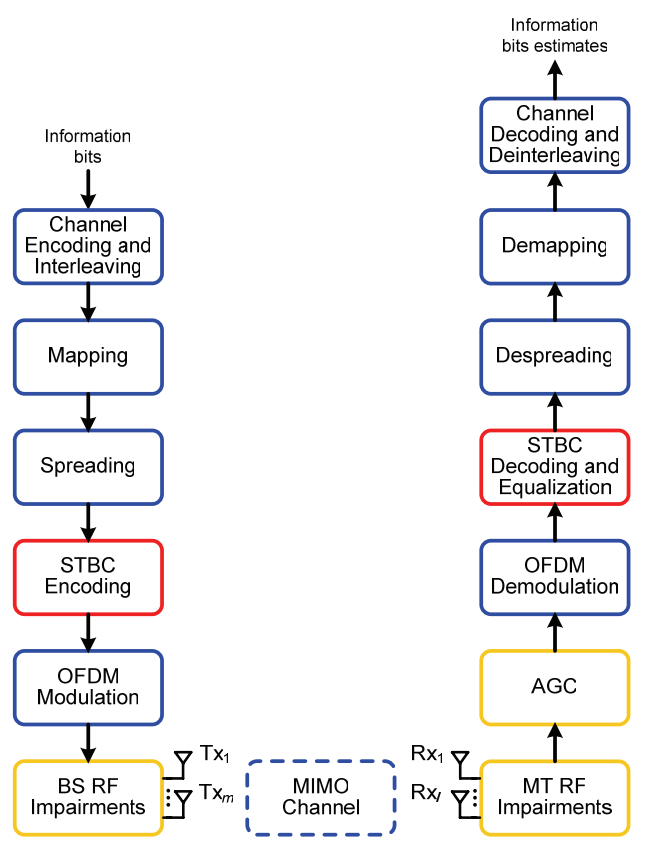

Figure 1. System model simulation chain 
Both base station (BS) and mobile terminals (MT) are considered to have an antenna array. The MIMO channel between BS and MT can be represented by matrix

$$
\boldsymbol{H}_{k}=\left[\begin{array}{ccc}
h_{1,1} & \cdots & h_{1, L} \\
\vdots & \ddots & \vdots \\
h_{M, 1} & \cdots & h_{M, L}
\end{array}\right],
$$

where $h_{m, l}$ represents the response of the existing channel between transmit antenna $m$, at the BS, and receive antenna $l$, at the considered $\mathrm{MT}_{k}$.

It is known that the spacing between elements in an antenna array has direct impact on the level of correlation between the channels associated to each of those antenna elements. It is reasonable to assume nearly independent fading processes when that distance is sufficiently large, justifying the hypothesis of very low correlation between versions of the same signal, carried through different channels, therefore making them quite useful for combined detection.

At the transmitter side, for each active $\mathrm{MT}_{k}$ in the system, the BS processes a stream of information bits, which after channel encoding and interleaving are mapped into complex QPSK symbols. Each of these is then spread into $S_{F}$ chips using the orthogonal WalshHadamard code set. All MT's chips are summed up before performing STBC encoding. The STBC coding process is explained in detail on section 3. After that, in the OFDM module, each resulting chip modulates one of the system's $N_{c}$ subcarriers, originating an OFDM symbol for each $P$ QPSK symbols $\left(P=N_{c} / S_{F}\right)$.

In order to take into account some RF impairments typical of real life systems, an RF module is considered both at the BS and at the MT. Besides that, an AGC module is also considered at the MT.

At reception, after $\mathrm{AGC}$, all operations that follow address each process step performed at the BS, in order to reach an estimate of the transmitted information bits. The STBC decoding module also includes the required equalization operation in order to deal with the fact that chips related with the same QPSK symbol were submitted to dissimilar channel fading as they were transmitted over distinct subcarriers.

\section{SPACE-TIME CODING SCHEMES}

In this section we present three space-time coding schemes, all based on the well known Alamouti STBC, which were implemented in the considered system model. The performance achieved by the system while using each of these schemes is presented in section 4 .

\subsection{Standard Alamouti $2 \times 1$}

Very briefly, in this well known scheme the encoder takes a block of two QPSK symbols, $s_{1}$ and $s_{2}$, in each encoding operation and forwards it to the transmit antennas according to the code matrix

$$
\boldsymbol{A}_{S}=\left[\begin{array}{cc}
s_{1} & -s_{2}^{*} \\
s_{2} & s_{1}^{*}
\end{array}\right],
$$

where the first column represents the first transmission period and the second column the second transmission period. The first and second rows correspond to the symbols transmitted from the first and second antenna, respectively. Transmitted $s_{I}{ }^{*}$ is the complex conjugate of symbol $s_{1}$.

In this scheme we assume one single reception antenna at the MT. The received signals, for each transmission period, are given by

$$
\begin{aligned}
& r_{1}=h_{1} s_{1}+h_{2} s_{2}+n_{1} \\
& r_{2}=-h_{1} s_{2}^{*}+h_{2} s_{1}^{*}+n_{2},
\end{aligned}
$$

where $h_{m}$ is the response of the channel between transmit antenna $m$ and the receiver antenna, assuming these channels are constant during two consecutive symbol transmission periods. Additive white Gaussian noise (AWGN) is represented by $n_{1}$ and $n_{2}$, which are independent complex random variables with zero mean and unit variance, relative to the first and second transmission periods, respectively.

In order to get the transmitted symbols estimates the received signals, on Eq. 3, are combined at the STBC decoder, in the following way:

$$
\begin{aligned}
& \widetilde{s}_{1}=h_{1}^{*} r_{1}+h_{2} r_{2}^{*} \\
& \widetilde{s}_{2}=h_{2}^{*} r_{1}-h_{1} r_{2}^{*}
\end{aligned}
$$

\subsection{Alamouti $2 \times 2$}

This scheme differs from the previous one only by the fact that the receiver has a pair of antennas, which offers the possibility to explore receive diversity, in addition to transmit diversity from which the previous scheme also benefits. In this case Eqs. 3 and 4 must take into account both receive antennas, and therefore they can be rewritten as follows, considering each receive antenna $l$ :

$$
\begin{aligned}
& r_{1, l}=h_{1, l} s_{1}+h_{2, l} s_{2}+n_{1} \\
& r_{2, l}=-h_{1, l} s_{2}^{*}+h_{2, l} s_{1}^{*}+n_{2}{ }^{\prime} \\
& \widetilde{s}_{1}=\frac{1}{2} \sum_{l=1}^{2}\left(h_{1, l}^{*} r_{1, l}+h_{2, l} r_{2, l}^{*}\right) \\
& \tilde{s}_{2}=\frac{1}{2} \sum_{l=1}^{2}\left(h_{2, l}^{*} r_{1, l}-h_{1, l} r_{2, l}^{*}\right)
\end{aligned}
$$




\subsection{Double Alamouti $4 \times 2$}

The proposed Double Alamouti scheme, represented in Fig. 2, consists on sending four QPSK symbols over four transmit antennas, in two transmission periods as the other two schemes presented here, according to the following code matrix:

$$
\boldsymbol{A}_{D}=\left[\begin{array}{cc}
s_{1} & -s_{2}^{*} \\
s_{2} & s_{1}^{*} \\
s_{3} & -s_{4}^{*} \\
s_{4} & s_{3}^{*}
\end{array}\right]
$$

In this scheme it is also considered that the channel behavior is constant over the two symbol periods. The received signals on each receiver antenna are given by the following expressions:

$$
\begin{aligned}
& r_{1, l}=h_{1, l} s_{1}+h_{2, l} s_{2}+h_{3, l} s_{3}+h_{4, l} s_{4}+n_{1} \\
& r_{2, l}=-h_{1, l} s_{2}^{*}+h_{2, l} s_{1}^{*}-h_{3, l} s_{4}^{*}+h_{4, l} s_{3}^{*}+n_{2}
\end{aligned} .
$$

In order to describe the implemented decoding approach for the required combination of the four received signals, we can write Eq. 8 in matrix form

$$
\left[\begin{array}{cc}
r_{1,1} & r_{1,2} \\
r_{2,1} & r_{2,2}
\end{array}\right]=\left[\begin{array}{cccc}
s_{1} & s_{2} & s_{3} & s_{4} \\
-s_{2}^{*} & s_{1}^{*} & -s_{4}^{*} & s_{3}^{*}
\end{array}\right] \cdot\left[\begin{array}{ll}
h_{1,1} & h_{1,2} \\
h_{2,1} & h_{2,2} \\
h_{3,1} & h_{3,2} \\
h_{4,1} & h_{4,2}
\end{array}\right]+\left[\begin{array}{cc}
n_{1,1} & n_{1,2} \\
n_{2,1} & n_{2,2}
\end{array}\right],
$$

where $r_{t, l}$ represents the received signal on transmission period $t$, at receive antenna $l$. For signal decoding, we can rewrite Eq. 9 in order to be able to estimate the transmitted symbols:

$$
\begin{aligned}
& {\left[\begin{array}{c}
r_{1,1} \\
r_{1,2} \\
r_{2,1}^{*} \\
r_{2,2}^{*}
\end{array}\right]=\left[\begin{array}{cccc}
h_{1,1} & h_{2,1} & h_{3,1} & h_{4,1} \\
h_{1,2} & h_{2,2} & h_{3,2} & h_{4,2} \\
h_{2,1}^{*} & -h_{1,1}^{*} & h_{4,1}^{*} & -h_{3,1}^{*} \\
h_{2,2}^{*} & -h_{1,2}^{*} & h_{4,2}^{*} & -h_{3,2}^{*}
\end{array}\right] \cdot\left[\begin{array}{c}
s_{1} \\
s_{2} \\
s_{3} \\
s_{4}
\end{array}\right]+\left[\begin{array}{c}
n_{1,1} \\
n_{1,2} \\
n_{2,1}^{*} \\
n_{2,2}^{*}
\end{array}\right] \Leftrightarrow .} \\
& \Leftrightarrow \boldsymbol{R}^{\prime}=\boldsymbol{H}^{\prime} \cdot \boldsymbol{S}+\boldsymbol{N}^{\prime}
\end{aligned}
$$

From Eq. 10, if $\boldsymbol{H}^{\prime}$ matrix is invertible it is easy to estimate the $\boldsymbol{S}$ transmitted symbols. For that, we can write the following expression based on the MMSE (Minimum Mean Square Error) criteria, in order to estimate the transmitted symbols

$$
\widetilde{S}=\boldsymbol{H}^{\prime \boldsymbol{H}}\left(\boldsymbol{H}^{\prime} . \boldsymbol{H}^{\prime \boldsymbol{H}}+\sigma_{c}^{2} . \boldsymbol{I}\right)^{-1} \boldsymbol{R}^{\prime}
$$

where $\sigma_{c}^{2}$ is the noise variance per sub-carrier, $\boldsymbol{I}$ is the identity matrix $(4 \times 4)$, and $(.)^{H}$ stands for the Hermitian operator (conjugate transpose).

After the decoding operation it is still necessary to perform symbol equalization given that the chips, referring to each transmitted symbol, were each exposed to dissimilar conditions as the propagation channel is not flat-fading. Filtering is therefore necessary to equalize the received OFDM signal. In this work we implemented a non-linear equalization based on MRC (Maximum Ratio Combining).

Considering a $\boldsymbol{W}$ matrix (4x4), such that

$$
\boldsymbol{W}=\boldsymbol{T} \cdot \boldsymbol{H}^{\prime},
$$

where $\boldsymbol{H}^{\prime}$ is the channel matrix represented earlier, and $\boldsymbol{T}$ represents the transformation matrix used for signal decoding, as in Eq. 11,

$$
\boldsymbol{T}=\boldsymbol{H}^{\boldsymbol{H}^{\boldsymbol{H}}}\left(\boldsymbol{H}^{\prime} . \boldsymbol{H}^{\boldsymbol{H}^{H}}+\sigma_{c}^{2} . \boldsymbol{I}\right)^{-\boldsymbol{I}}
$$

The equalization coefficients are determined for each subcarrier since processing is done at the chip level. The weights to be applied to the signal for MRC equalization are

$$
g_{j}=\frac{w_{j j}^{*}}{\sigma_{c T_{j}}}
$$

where $w_{j j}, j=1, \ldots, 4$, represents the diagonal elements of the $\boldsymbol{W}$ matrix, whose chips correspond respectively to the four symbols in estimation. This equalization method needs information regarding noise and therefore $\sigma_{c T j}$ represents in the latter expression the total noise standard deviation per subcarrier regarding the chip belonging to transmitted symbol $s_{j}$. The total noise

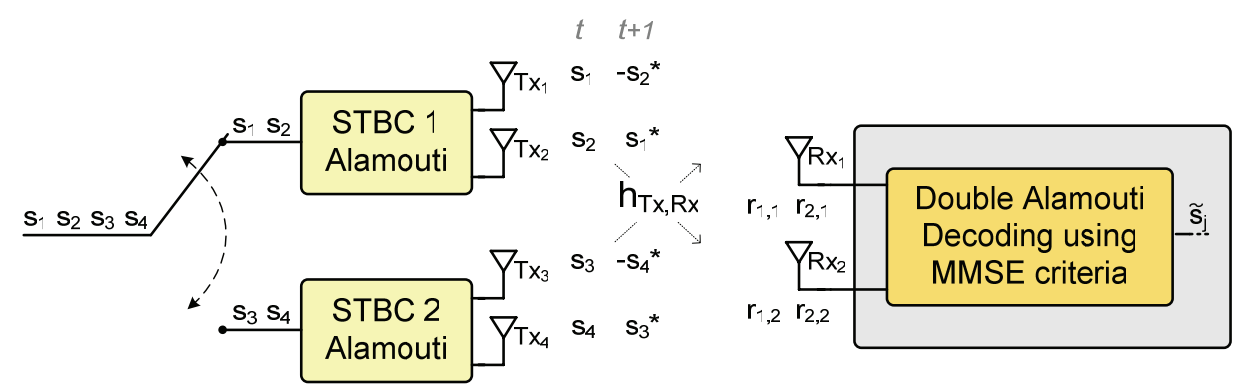

Figure 2. Double Alamouti coding scheme 
variance per subcarrier is given by

$$
\sigma_{c T j}^{2}=\sigma_{c}^{2} \sum_{i=1}^{4}\left|t_{j i}\right|^{2}+\sum_{\substack{i=1 \\ i \neq j}}^{4}\left|w_{j i}\right|^{2}+(K-1) \sum_{i=1}^{4}\left|w_{j i}\right|^{2},
$$

where the first term stands for the noise variance, the second term relates with ISI (Inter-Symbol Interference) variance, and the last term refers to MAI (Multiple Access Interference) variance, assuming that mapping constellation is normalized, and $K$ is the number of active users in the system.

For each QPSK symbol it is also necessary to perform a normalization of the equalization weights:

$$
g_{f} \times \frac{S_{F}}{\sum_{f=1}^{S_{F}} \frac{\left|w_{f f}^{\prime}\right|^{2}}{\sigma_{c T f}}},
$$

where $f$ is the chip index of one specific QPSK symbol in estimation, associated to the $S_{F}$ size $\boldsymbol{W}$ ' matrix.

\section{NUMERICAL RESULTS}

In order to evaluate the performance of the proposed Double Alamouti space-time coding scheme, we used a MIMO channel derived from the system level channel model specified by the 3GPP/3GPP2 SCM AHG $\left(3^{\text {rd }}\right.$ Generation Partnership Project Spatial Channel Model Ad-Hoc Group) [6]. Our channel is mainly an adaptation at $5 \mathrm{GHz}$ of the 3GPP model and is generated in two steps. The first one generates slow fading parameters and is performed before running the actual simulation chain. It produces data containing spatial ray description of the channel for a given realization of slowly varying parameters, which is used afterwards in the second step to generate the corresponding fast fading channel response. In the first step, time related parameters were derived from the ETSI BRAN E channel model defined in the European BRAN Hiperlan/2 standardization project [7]. The 3GPP Urban Macro scenario was considered regarding spatial parameters.

For this work we considered that the transmission system operates only in the downlink (DL), and uses Walsh-Hadamard spreading sequences of length 32 . The system exploits only 672 subcarriers from a total of 1024 subcarriers, while the remaining ones are used as guard subcarriers. The guard interval between each OFDM symbol is a cyclic prefix with duration of about $19 \%$ of the OFDM symbol duration. Perfect channel estimation was assumed, and the channel was considered to be constant during two OFDM symbols interval $(\approx 43 \mu \mathrm{s})$. A summary of the most important parameters settings used in our computer simulations is presented in Tab. 1 .

\begin{tabular}{|c|c|c|}
\hline \multicolumn{2}{|c|}{ Carrier Frequency } & $5.2 \mathrm{GHz}$ \\
\hline \multicolumn{2}{|c|}{ Bandwidth } & $61.44 \mathrm{MHz}$ \\
\hline \multicolumn{2}{|c|}{ Spreading Factor $\left(S_{F}\right)$} & 32 \\
\hline \multicolumn{2}{|c|}{ Num. of Subcarriers $\left(N_{c}\right)$} & $672(1024)$ \\
\hline \multicolumn{2}{|c|}{ OFDM Symbol Duration } & $21.51 \mu \mathrm{s}$ \\
\hline \multicolumn{2}{|c|}{ Guard Period } & $4.16 \mu \mathrm{s}$ \\
\hline \multicolumn{2}{|c|}{ Antennas Spacing } & $10 \lambda(\mathrm{BS}), \quad \lambda / 2(\mathrm{MT})$ \\
\hline \multicolumn{2}{|c|}{ Antenna Elements } & $2 \times 1,2 \times 2,4 \times 2$ \\
\hline \multicolumn{2}{|c|}{ Modulation $\left(N_{b}\right)$} & 16-QAM (4), QPSK (2) \\
\hline \multicolumn{2}{|c|}{ Coding } & UMTS CC $\left(R_{c}=1 / 2\right)$ \\
\hline \multicolumn{2}{|c|}{ Info. Bit Rate (per code) } & $3.3 \mathrm{Mbps}$ \\
\hline \multicolumn{2}{|c|}{ Mobile Terminal Speed } & $60 \mathrm{~km} / \mathrm{h}$ \\
\hline \multirow{2}{*}{$\begin{array}{c}\text { Channel } \\
\text { Model }\end{array}$} & Spatial & 3GPP Urban Macro \\
\hline & Time & ETSI BRAN E \\
\hline
\end{tabular}

Table 1: Parameters settings in simulations

The system uses convolutional channel coding based on the UMTS code with coding rate $R_{c}=1 / 2$, as well as interleaving also based on UMTS specifications. The antennas spacing at the base station is $10 \lambda$ and is $\lambda / 2$ at the mobile terminals.

In our simulations, the noise variance per carrier is calculated according with the expression

$$
\sigma_{c}^{2}=\frac{10^{-\frac{E_{b} / N_{0}}{10}}}{N_{b} R_{c}} \frac{S_{F}}{K},
$$

where $N_{b}$ is the number of bits mapped into one modulated symbol.

We present the performance results of the considered system, achieved by all three space-time coding schemes presented in section 3. In order to allow an appropriate comparison of these schemes, the same spectral efficiency was considered for the three implementations. Therefore, 16-QAM modulation was used when the system implemented the two coding schemes based on standard Alamouti, while QPSK was used together with the Double Alamouti coding scheme. In short, for the latter configuration four QPSK data symbols are transmitted at a time, while for the other two schemes only two 16-QAM symbols are transmitted in the same time interval, thus offering the same spectral efficiency.

The performance results achieved by the considered system, through all three coding schemes are represented by the curves drawn in Fig. 3, according with $E_{b} / N_{0}$. All results were attained considering a fully loaded system $(K=32)$. In this graphical representation is quite visible the gain offered by the antenna array with two elements, integrated at the mobile terminals, as the performance delivered by the single Alamouti $2 \times 1$ scheme is almost $5 \mathrm{~dB}$ worse than the one achieved by any of the two other coding schemes. It is also clear that the results attained with the $2 \times 2$ and $4 \times 2$ schemes are 


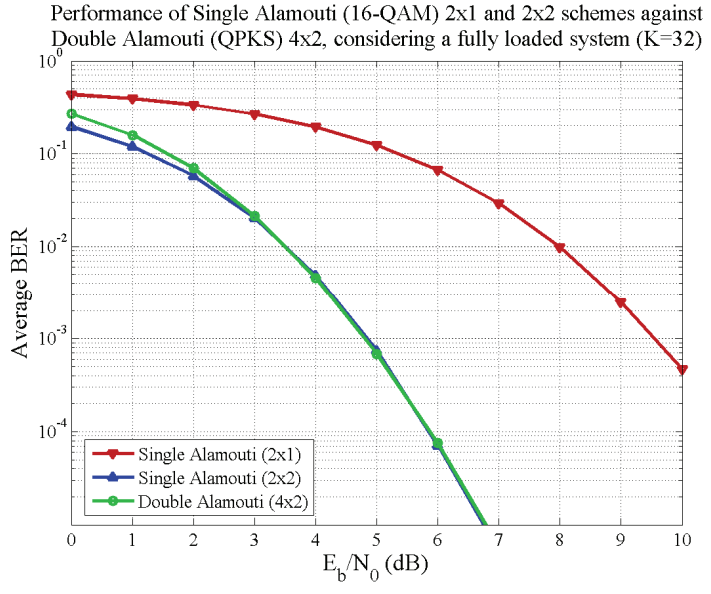

Figure 3. Performance results

almost alike, for the considered system without any particular impairment (RF or AGC, i.e. yellow outlined blocks in Fig. 1).

In order to evaluate the schemes presented here, in a more realistic scenario, we also simulated this same system while considering typical RF impairments at both ends of the transmission chain, as well as an AGC block at the mobile terminal. These blocks which model some impairments found in real transmission systems were designed and configured according to typical implementations in real systems [1]. Performance results considering these system impairments are shown in Fig. 4.

As expected, the Double Alamouti $4 \times 2$ scheme provides better tolerance to phase and amplitude errors arising from nonlinearities typical of practical implementations of transmission systems, since QPSK modulation is less sensitive to these issues, when compared with 16-QAM.

\section{CONCLUSION}

In this paper we evaluated an extended space-time coding technique based on the popular Alamouti scheme, for MC-CDMA systems. The proposed Double Alamouti scheme should be an interesting option when its extra complexity is not excessive for practical implementations. When compared against single Alamouti, it allows reducing the modulation order by a factor of two while preserving bandwidth efficiency, i.e. the throughput achieved with the combination of single Alamouti and 16-QAM modulation can be reached with Double Alamouti combined with QPSK modulation, without sacrificing spectral efficiency. The order reduction of the modulation scheme allows using modulations that are more tolerant to impairments like phase or frequency errors, or nonlinear distortion.

\section{ACKNOWLEDGMENTS}

This work was partially supported by European project IST-2002-507039 (4MORE)

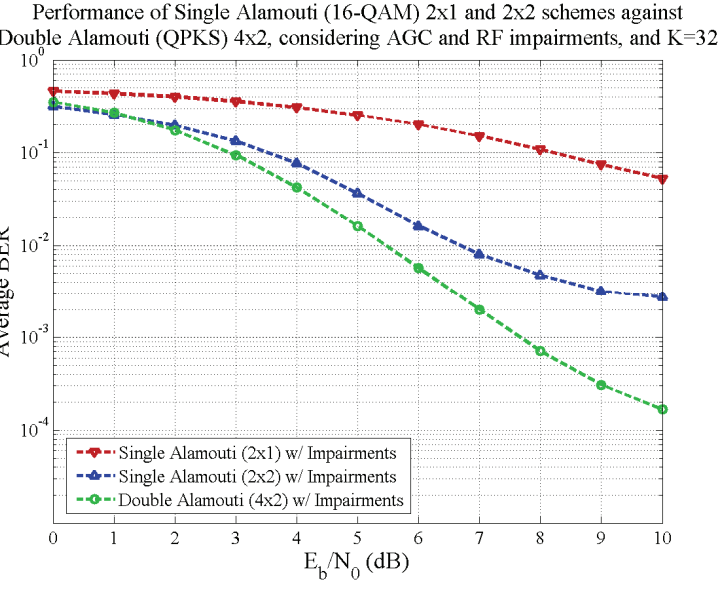

Figure 4. Performance results, considering impairments

\section{REFERENCES}

[1] IST 4MORE project web site http://www.ist-4more.org (June 2006)

[2] R. Prasad, S. Hara, "An overview of multi-carrier CDMA,” Proc. IEEE ISSSTA'96, Sept. 1996, pp. 107-114.

[3] N. Yee, J.-P. Linnartz, G. Fettweis, "Multi-carrier CDMA in indoor wireless radio networks," Proc. IEEE PIMRC'93, Sept. 1993, pp. 109-113.

[4] W. H. Gerstacker, F. Obernosterer, R. Schober, A. Lehmann, A. Lampe, and P. Gunreben, "Widely Linear Equalization for Space-Time Block-Coded Transmissions Over Fading ISI Channels," Proc. of IEEE International Conference, 2000, pp. 238-240.

[5] S. M. Alamouti, "A simple transmit diversity technique for wireless communications," IEEE Journal on Selected Areas in Communications, vol. 16, no. 8. pp. 1451-1458, Oct. 98.

[6] 3GPP TR 25.996 v.1.0.0 m (2003-05) - $3^{\text {rd }}$ Generation Partnership Project; Technical Specification Group Radio Access Network; Spatial channel model for MIMO simulations (Release 6)

[7] J. Medbo, "Channel Models for Hiperlan/2 in different Indoor Scenarios," ETSI BRAN doc. 3ERI085b, 1998 